\title{
Editorials
}

\section{LEPROSY-LIKE DISEASE OCCURRING NATURALLY IN ARMADILLOS}

The most important advances in our knowledge of leprosy in the past 15 years have stemmed from studies on experimental infections with Mycobacterium leprae in animals. First by Shepard (1960) based on limited infection in the mouse following foot pad inoculation and later by Kirchheimer and Storrs (1971) based on disscminated infection in the 9-banded armadillo (Dasypus novemcinctus) following inoculation with $M$. leprae. While both animal models continue to be exploited, and the readily available and laboratory bred mouse is being used throughout the world, the armadillo is mainly used where the species is indigenous and entirely on animals caught from the wild because they fail to breed in captivity. The special importance of the armadillo model is as the sole source of large quantities of $M$. leprae since the organism has not been grown in vitro. It is against this background therefore that the recent discovery by Walsh et al. (1975) of a leprosy-like disease occurring naturally in armadillos trapped in South West Louisiana has caused so much concern and interest. Their research leading up to this discovery was carried out by Gulf South Research Institute (GSRI), Louisiana, which was where in collaboration with the Public Health Service Hospital, also in Louisiana, the original studies on the armadillo model were undertaken.

The discovery by Walsh and his colleagues was made during studies on naturally occurring diseases among armadillos captured from the wild in South West Louisiana. In their 1975 publication they reported 7 animals with large numbers of acid-fast bacilli in skin lesions, nerves, lymph nodes, spleens and livers at the time of necropsy and when the animals had been housed from 1 day to 15 weeks in the outer holding area at GSRI. The histology of the infected tissues and the distribution of bacilli therein was typical of lepromatous leprosy in man and identical with the picture seen in armadillos with diffuse acid-fast bacillary infection following experimental inoculation with $M$. leprae from man. Bacilli from 4 of the animals so far cultured for 3 months, failed to grow on conventional media for the cultivation of mycobacteria incubated at $32^{\circ}$ or $37^{\circ} \mathrm{C}$. While the authors drew attention to the resemblance of the AFB isolates to $M$. leprae they referred to other important criteria which were being investigated and their continuing study of the prevalence and distribution of natural leprosy-like disease in armadillos in Louisiana. Already some of these results have recently been published as well as the experience of others equally concerned with and experienced in studies on armadillos from Louisiana for leprosy research. GSRI have now identified 14 naturally infected armadillos trapped 17 to 39 miles from their Institute, representing approximately $10 \%$ of uninoculated animals, of which only 2 had been in captivity for more than 5 months before examination (reported in Morbidity and Mortality Weekly Report, 1976). 
Furthermore, from the patterns of Mitsuda reactivity in patients with leprosy and loss of acid-fast staining following pyridine extraction of bacilli isolated from armadillos infected in the wild strongly suggest that they are $M$. le'prae (Walsh e't al., 1976; Morbidity and Mortality Weekly Report, 1976), although their final identification cannot be considered definite. On the other hand, Kirchheimer (1976) at the Public Health Service Hospital, Carville, in South East Louisiana has found no evidence whatsoever of mycobacteriosis in 133 wild armadillos captured within this area of Louisiana or from 87 and 13 similarly captured armadillos from Florida and Texas, respectively.

This new and unexpected data from GSRI has obviously generated deep concern and interest since it indicates for the first time that there could be a non-human source of $M$. leprae (i.e. the armadillo) and therefore an important public health risk for those regions in the world where this animal species is indigenous, but at the same time challenge and possibly jeopardise future advances in leprosy research dependent upon the armadillo model.

Assuming armadillos in the wild in Louisiana are infected with $M$. leprae, is there any evidence that they are a source for infecting man? Epidemiological data does not support such evidence, since leprosy has been endemic in the resident population of Louisiana for 150 years, whereas the 9-banded armadillo has been there only since 1926 (entering then from Texas and since spreading eastwards as far as (ieorgia and Florida), and in the past 50 years the incidence of leprosy has been decreasing in Louisiana. Moreover, a recently conducted case control study undertaken by the Centre for Disease Control, Atlanta, on leprosy patients reported from Louisiana since 1966 who had no family history of leprosy, showed no greater contact with armadillos than did matched controls (Morbidity and Mortality Weekly Report, 1976).

Thus with no evidence to implicate the armadillo as a source of infecting man with leprosy in Louisiana, what are the implications of the GSRI findings on the use of the armadillo for leprosy research? They could jeopardise the whole future of this important programme. However, because of the importance of the armadillo in providing vast quantities of $M$. leprae, it is to be hoped that no hasty decisions will be made. The data must be used as a timely reminder of the extra precautions which have to be taken for studies entirely dependent on animals caught from the wild. On the basis of GSRI data that $10 \%$ of wild armadillos have a spontaneous $M$. leprae-like infection, there are sceptics who challenge whether any infections with $M$. leprae have been transmitted experimentally to armadillos. This extreme view can be discounted, since the incidence in experimentally inoculated armadillos is at least 50\%. Also 16 armadillos caught from the wild, with no evidence of gross myobacteriosis, were shipped to us in the UK by GSRI. They were inoculated intravenously here with $M$. leprae from man, and by the end of a year 9 had gross evidence of multiple skin lesions, 8 of which had a skin nodule at the site of the intravenous inoculation.

Nevertheless, in future, detailed procedures for detecting every type of mycobacterium must be used for screening wild armadillos. They must then be held in quarantine for at least 3 months before being finally inoculated with M. leprae. Furthermore, at present stocks of experimentally infected armadillos must be inoculated with $M$. leprae from man and not with serially passaged organisms. Although no cultivable species of mycobacteria have so far been isolated from armadillos in Louisiana, Muñoz Rivas (1973) has isolated cultivable strains of $M$. avium and $M$. intracellulare from some 9-banded armadillos caught 
from the wild in Colombia, South America. The world-wide distribution of M. lepraemurium among wild rats might well, under the environmental conditions in which armadillos live, result in such an infection. However, apparently 9-banded armadillos failed to develop infection inoculated with $M$. lepraemurium (Dr J. Convit, personal communication).

There still remains to be clarified the finding by GSRI of M. leprae-like infections in wild armadillos. If further studies on these isolates confirm their identity as M. leprae, what is their source? Since within Louisiana, GSRI and Carville are the only centres inoculating large numbers of armadillos with $M$. leprae, and GSRI but not Carville are reporting $M$. leprae-like infections in uninoculated animals caught from the wild, a possible source could be the spread of $M$. leprae from experimentally infected armadillos at GSRI. However, of the 14 armadillos with $M$. leprac-like infection, only 2 had been more than 5 months in captivity at GSRI before manifesting overt disease. Furthermore, all the armadillos had been trapped from locations 17 to 39 miles from GSRI and at locations 18 to 44 miles between each other, whereas the home range of the adult armadillo is believed to be only 8 to 10 acres. While Issar (1976) reported a massive $M$. leprae-like infection in an uninoculated armadillo in 1973, that had been maintained for 2 years in GSRI's outer animal compound, GSRI claim that their discovery was only made since 1974. Because of the importance of these claims, with their varying discrepancies, the only way to clarify them is by a full investigation. This can be achieved by an independent and detailed survey of mycobacteriosis among randomly captured armadillos in Louisiana. Fortunately the Center for Disease Control, Atlanta, are currently undertaking such a survey covering 600 armadillos, and the results of their investigations are eagerly awaited.

\section{References}

Issar, S. L. (1976). Leprosy Scientific Memoranda L-778/1.

Kirchheimer, W. F. (1976). Leprosy Scientific Memoranda L-772/1.

Kirchheimer, W. F. and Storrs, E. E. (1971). Int. J. Lepr. 39, 693.

Morbidity and Mortality Weekly Report (DHEW Publication No. (CDC) 76-8017). (1976). 25, 18.

Muñoz Rivas, G. (1973). Revta Sälud Públ., Lima 33, 61.

Shepard, C. C. (1960). J. exp. Med. 112, 445.

Walsh, G. P., Storrs, E. E. Burchfield, H. P., Cottrell, E. H., Vidrine, M. F. and Binford, C. H. (1975).

L-771/1. 